Original Paper http://ajol.info/index.php/ijbcs ～http://indexmedicus.afro.who.int

\title{
Examen de la gestion et stratégies de protection des berges du Lac Bam à Kongoussi au Centre-Nord du Burkina Faso
}

\author{
Karim DRABO, Joséphine YAMEOGO* et Louis SAWADOGO \\ Université Senghor, Campus de Ouagadougou, DEF/INERA/CNRST, \\ 03 BP 7047 Ouagadougou 03, Burkina Faso. \\ *Auteur correspondant; E-mail : finayame@yahoo.fr
}

\section{REMERCIEMENTS}

Les auteurs remercient les anciens députés du Groupe Parlementaire Alternance-Démocratie et Justice (ADJ) de l'Assemblée Nationale et le président de la Commission du Développement Economique et de l'Environnement (CODE) pour leurs soutiens financiers et multiformes dans la réalisation de ce travail.

\section{RESUME}

La dégradation du lac Bam au Burkina Faso préoccupe les acteurs nationaux et internationaux. Le présent travail vise principalement à contribuer à la connaissance des facteurs de dégradation et des stratégies de gestion durable. Spécifiquement, il voudrait identifier les acteurs impliqués dans la gestion des berges ; examiner les raisons ou les causes de dégradation; identifier les stratégies de restauration. Alors, des recherches bibliographiques et des interviews ont été réalisées dans cinq villages riverains, auprès de cent quarante producteurs; sept ONG et Associations, trois services publics déconcentrés, un service de la collectivité territoriale et un chef coutumier. Les résultats révèlent des mérites et des insuffisances dans les missions assignées aux acteurs. La principale raison de l'occupation des berges demeure la quête permanente de la ressource en eau. Les sécheresses, les mauvaises pratiques culturales, la déforestation et les problèmes démographiques sont des causes de dégradation caractérisées par des indices de sévérités importants allant de 1,1 à 1,4. Les producteurs proposent quatre alternatives à l'occupation des berges. Sept points de réflexion sont également exposés. Toutefois, le succès des stratégies repose sur une volonté de changement chez les producteurs et les moyens matériels et financiers ainsi que l'identification de circuits d'écoulement des produits.

() 2016 International Formulae Group. All rights reserved

Mots clés: Lac Bam, dégradation, facteurs, berges, stratégies de protection.

\section{Management evaluation and protection strategies of Lake Bam riverbanks at Kongoussi in the North-Central Burkina Faso}

\begin{abstract}
The degradation of the Lake Bam in Burkina Faso is what worries the national and international actors. The present work mainly aims at contributing to the knowledge of the causes of degradation and sustainable management strategies. Specifically, it would identify the actors involved in the management of the riverbanks;
\end{abstract}


examine the reasons or the causes of degradation; identify restoration strategies. So, literature searches and interviews were conducted in five lakeside villages, on one hundred forty producers; seven NGOs and Associations, three decentralized public services, one service of the territorial collectivity and one tribal chief. The results show the merits and shortcomings of the mission assigned to the actors. The main reason of the occupation of the riverbanks remains the permanent search for water resource. Droughts, poor farming practices, deforestation and population issues are the causes of deterioration characterized by important indices of severity ranging from 1.1 to 1.4. The producers offer four alternatives to the occupation of the riverbanks. Seven points of thoughts are also exposed. However the success of the strategies is based on a desire for changing among producers; material and financial resources and the identification of flow paths of the products.

(C) 2016 International Formulae Group. All rights reserved

Keywords: Lake Bam, degradation, factors, banks, protection strategies.

\section{INTRODUCTION}

$\mathrm{Au}$ Burkina Faso, les trois grands écosystèmes sont terrestres $(91,22 \%)$, aquatiques $(0,26 \%)$ et intermédiaires $(1,34 \%)$. Le reste du territoire est essentiellement constitué de dunes, de rochers et des villes (Zoubga, 2007). Les écosystèmes aquatiques d'où relèvent les zones humides, bien que représentant une faible proportion sont d'une importance capitale en matière de conservation de la diversité biologique. Leur gestion s'inscrit dans un contexte international notamment la convention Ramsar dont la mission est la conservation et l'utilisation rationnelle des zones humides par des actions locales, régionales et nationales et par la coopération internationale, en tant que contribution à la réalisation du développement durable dans le monde entier (SC-Ramsar, 2013). Le Burkina Faso a inscrit une quinzaine de sites sur la liste des zones humides d'importance internationale de Ramsar. Celles-ci sont pourvoyeuses de biens et services écosystémiques à répercussions socioéconomiques perceptibles aux niveaux local, national et international. Elles sont toutes en proie aux activités humaines et subissent les aléas climatiques. Le Lac Bam qui est un site Ramsar est la plus importante étendue d'eau naturelle du Burkina Faso. Il est sujet à des facteurs sous toutes les formes qui menacent son existence comme constatés dans d'autres zones humides (Badahoui et al., 2009). La capacité de stockage de l'eau qui était initialement de 40 millions de $\mathrm{m}^{3}$ est de nos jours 37 millions de $\mathrm{m}^{3}$. Par conséquent le défi consiste à gérer ce lac dans une vision de durabilité afin de renforcer sa contribution à la sécurité alimentaire et à la lutte contre la pauvreté (MEDD, 2014). Le renforcement des actions de gestion durable de ce lac nécessite au préalable un examen des rôles des acteurs, une analyse des facteurs de dégradation, des stratégies de restauration et de protection des berges. Ainsi, le présent travail voudrait contribuer à la connaissance des facteurs de dégradation et des stratégies de gestion durable. Il vise spécifiquement à identifier les acteurs impliqués dans la gestion des berges ; examiner les causes ou les raisons de dégradation; identifier les stratégies de restauration.

\section{MATERIEL ET METHODES}

\section{Le site de l'étude}

Le site de l'étude est la province du Bam, en particulier le Lac Bam localisé dans la Région du Centre-Nord du Burkina Faso. La ville de Kongoussi, chef-lieu de la province, est située entre $1^{\circ} 30^{\prime}$ de longitude Ouest et $13^{\circ} 22^{\prime}$ de latitude nord. Le Lac Bam s'étend sur $35 \mathrm{~km}$ avec un bassin versant de 2 $610 \mathrm{~km}^{2}$. L'envasement du lac est d'environ 4,8 millions de $\mathrm{m}^{3}$ (Drabo, 2015). La pluviométrie moyenne annuelle est de l'ordre de $699 \mathrm{~mm}$ avec des températures moyennes maximales de l'ordre de $42{ }^{\circ} \mathrm{C}$. Les sols sont majoritairement argilo-sableux. La végétation 
est à dominance rabougrie, épineuse et aussi des espèces utilitaires arborescentes telles que Vitellaria paradoxa, Adansonia digitata (Thiombiano et al., 2010) ). On retrouve également des espèces comme Commiphora africana, Grewia flavescens et Pterocarpus lucens. Les herbacées sont essentiellement les graminées des genres Pennisetum, Andropogon et de la famille des Cyperaceae (Sawadogo, 2005).

\section{Échantillonnage et collecte des données}

Cinq (05) villages riverains ont été concernés par l'étude, dont trois (03) situés sur la rive droite. Parmi ces trois villages, Kora et Darigma ont une forte activité maraîchère; Tanguiema a une faible activité maraîchère. Les deux (02) autres villages Pouni et Bam sont situés sur la rive gauche avec une faible activité maraîchère. L'échantillon était constitué de quarante (40) producteurs à Kora, quarante (40) producteurs à Darigma et vingt (20) producteurs pour chacun des trois autres villages à faible activité maraîchère. Ce qui donne un échantillon de cent quarante (140) producteurs interrogés (cible 1). Trois techniques de collecte de données ont été combinées : l'interview semi-structurée à l'aide d'un guide d'entretien adressé aux acteurs au niveau local, d'un questionnaire adressé aux exploitants situés sur les berges du lac et l'observation directe. Un guide d'entretien a été administré aux responsables des services administratifs et techniques de l'Etat, des ONG et Associations ainsi que des Coopératives (cible 2). Il s'agit de douze (12) acteurs locaux composés de sept (07) ONG et Associations, trois (03) services déconcentrés de l'administration, un (01) service de la collectivité territoriale et une (01) personne ressource chef coutumier. Les observations directes sur l'état, l'occupation des berges ont permis de disposer d'illustrations photographiques durant la période de l'étude.

\section{Analyse des données}

Les données quantitatives recueillies ont été traitées avec le logiciel SPSS. L'analyse a globalement consisté à considérer les opinions de tous les enquêtés par rapport à chaque indicateur recherché par cette étude. L'indice de sévérité a été calculé de manière participative selon la méthode de Smith et al. (2001) :

Indice de sévérité $\mathrm{s}=1+(\mathrm{r}-1) /(\mathrm{n}-1)$

$\mathrm{r}=$ rang de la menace (par ordre d'importance selon le participant); $\mathrm{n}=$ nombre total des menaces énumérées par le participant. $\mathrm{S}$ considérée pour une menace donnée est la moyenne des sévérités chez tous les participants qui ont listé cette menace. $1 \leq \mathrm{S} \leq 2$. Une faible valeur de $\mathrm{S}$ traduit une grande sévérité.

\section{RESULTATS}

\section{Les acteurs locaux de l'occupation des berges du Lac Bam}

Le Tableau 1 présente ces acteurs, leurs missions, leurs mérites et insuffisances. Les principaux exploitants sont les populations riveraines et les personnes ressources comme les chefs coutumiers; les agents des services techniques déconcentrés de l'Etat; les membres des Associations et des groupements des usagers du lac; Les Organisations de la Société Civile (OSC); les Collectivités Territoriales (CT). Les activités pratiquées sont du ressort de l'agro-sylvo-pastoralisme et halieutiques.

\section{Les raisons de l'occupation des berges du Lac Bam}

Il ressort des investigations que les raisons de l'occupation des berges sont toutes liées aux activités agricoles et directement ou indirectement à la ressource en eau. Ces raisons peuvent être classées en quatre groupes comme l'indique la Figure 1 :

- la proximité de l'eau : une proportion de $81,4 \%$ des exploitants des berges en majorité des agriculteurs-maraîchers avancent cette raison justifiée par les changements climatiques qui se traduisent par des baisses 
de pluviométries, la diminution significative du nombre de pluies par an, les poches de sècheresse.

- la fertilité des sols: les pressions anthropiques entraînant la surexploitation des terres ainsi que les péjorations climatiques ont conduit $15 \%$ des producteurs à occuper les berges.

- l'insuffisance de terres cultivables: Pour $2,1 \%$ des producteurs interrogés, l'insuffisance de terres est notoire dans la province du Bam comme c'est le cas dans plusieurs localités du Burkina Faso. Elle est une raison de leur présence sur les berges

- diverses autres raisons : des raisons liées à des conflits fonciers, à des superficies aussi acquises gratuitement amènent une minorité à s'installer sur les berges.

\section{Les causes de la dégradation des berges du lac Bam}

Selon les personnes interrogées, les causes de la dégradation du Lac Bam peuvent être classées en deux groupes: les causes climatiques sont les sécheresses, l'évaporation accélérée de l'eau résultant des fortes températures. Les causes anthropiques concernent les mauvaises pratiques agricoles, la déforestation, la surexploitation des pâturages.

- Les sécheresses: A travers l'indice de sévérité calculé $(1,1)$, les populations reconnaissent que les sécheresses constituent la plus importante cause de la dégradation des berges du Lac Bam. Les effets se manifestent par l'aridité des sols et les baisses des performances des cultures annuelles et de contre-saison.

- Les mauvaises pratiques agricoles: Comme l'indique la sévérité $(1,2)$ les pratiques agricoles favorisent la dégradation des berges. Dans les sites de l'étude, l'agriculture est de type extensif sur des terres marginales, utilisant des moyens rudimentaires. Le travail du sol n'est pas fait dans le respect des prescriptions techniques pour le maintien de sa fertilité. A cette défaillance s'ajoute la mauvaise utilisation des pesticides et des herbicides, sources de pollution des terres et des eaux, les creusées de tranchées pour la canalisation de l'eau (Figure 2). Dans le même, sens il est reconnu que les motopompes de la majorité des producteurs sont vieillissantes et produisent des déchets d'huile et de gasoil polluant le sol, l'eau, l'air, donc les cultures. Les populations reconnaissent que la pratique de la jachère tend à disparaitre, compte tenu de la pression démographique.

- La déforestation: Avec une sévérité de 1,2 au même titre que les mauvaises pratiques agricoles, la déforestation constitue une cause de dégradation des berges. Les besoins en bois d'énergie suscitent des défrichements de terres qui dépassent les régénérations et les reboisements. Des espèces utilitaires comme Adansonia digitata, Vitellaria paradoxa, Balanites aegyptiaca et Lannea microcarpa d'intérêts énergétiques et artisanaux ont souvent subi des coupes clandestines.

- Les problèmes démographiques: caractérisés par une sévérité de 1,4, ils constituent une cause explicative des autres causes anthropiques de la dégradation des berges. En effet, les populations affirment que l'extension des terres cultivées, la déforestation, la surexploitation des pâturages et l'exercice d'activités génératrices de revenus sur les berges sont consécutives à une démographie croissante avec son cortège de besoins à satisfaire chez l'homme. On assiste à des phénomènes migratoires dans les villages riverains du lac surtout en saison sèche.

- La surexploitation des pâturages : le Lac Bam constitue la principale source d'abreuvement des animaux de la province, voire d'autres contrées, surtout en période de saison sèche. La conséquence directe de cette situation est le piétinement intensif des berges du lac et la dégradation de leur couvert végétal, d'où une sévérité de 1,5.

- Evaporation accélérée de l'eau : sans avoir une bonne connaissance des relevées de températures, les populations constatent que 
de nos jours l'eau du lac s'évapore rapidement sous l'effet de soleil brûlant et de l'ensablement du lac $(\mathrm{S}=1,5)$.

- Les activités rémunératrices non agricoles: En plus des activités agro-sylvopastorales et halieutiques, l'eau du lac est exploitée pour des activités génératrices de revenues $(S=1,6)$. Au nombre de ces activités on retrouve la briqueterie, les travaux liés à l'orpaillage. La Figure 3 présente une mauvaise gestion des berges du Lac Bam due à la fabrication de briques.

Les stratégies de restauration et de protection des berges du Lac Bam

L'adoption des techniques de protection et restauration des berges

L'étude a permis de connaître les différentes mesures de protection des sols et des ressources en eau vulgarisées par les services techniques, les associations et $\mathrm{ONG}$ compétents. Les domaines d'intervention de ces structures sont proportionnellement les sensibilisations à l'abandon de toute activité dans la bande de servitude $(70,7 \%)$; la plantation d'arbres $(22,9 \%)$; la mise en place de paquets technologiques notamment les cordons pierreux, le zai, le paillage $(4,3 \%)$; l'érection de bande enherbée $(1,4 \%)$ et autres mesures $(0,7 \%)$ tel que le traitement des ravines.

L'examen de l'adoption de ces techniques au sein des producteurs révèle que celles préférées et adoptées sont les techniques présentées par la Figure 4. Avec des taux d'adoption supérieurs à $60 \%$, les cordons pierreux et le paillage sont les plus prisées puis le zai. Si les deux premières sont qualifiées de moins coûteuses, la troisième est perçue comme une technique très laborieuse. Quant aux bandes enherbées, surtout aux haies vives les résultats parâ̂ssent moins immédiats que les trois premiers.

Le retrait de la bande de protection

Sur un total de cent quarante (140) exploitants interrogés, $87,1 \%$ ont connaissance de l'existence de la bande de servitude, contre 12,9\% qui l'ignorent. Cette population, en grande partie $(93,6 \%)$, ignore les textes qui régissent cette bande. Cela pourrait s'expliquer par l'analphabétisme chez $67,9 \%$ des producteurs. La non matérialisation de cette bande n'est pas sans conséquences néfastes. La plupart des maraîcher-culteurs n'ont pas de motopompes, ce qui rend pénible l'arrosage des cultures à distance et les amène à occuper les berges.

\section{Le regroupement des acteurs en associations de producteurs}

Les regroupements de producteurs déjà mentionnés dans le Tableau 1 se situent dans le contexte de la gestion participative des ressources naturelles prônées par les structures nationales et internationales. Comme indiqué au Tableau 1, les Associations et groupements des usagers du lac et les Organisations de la Société Civile (OSC) œuvrent dans des domaines et selon des missions précisément définies. On distingue les regroupements créés par l'initiative des populations et ceux créés par l'Etat ou ses partenaires. Sur les sites de l'étude, on retrouve les Comités Villageois de Gestion des Terroirs (CVGT) créés par le Programme National de Gestion des Terroirs (PNGT), programme qui a œuvré pour la mise en application de la Réforme Agraire Foncière (RAF). Parmi les plus importants regroupements travaillant en faveur du Lac Bam, on retient la Coopérative Maraîchère de Kongoussi (COOMAKO), la Société Coopérative du Lac Bam (SCOBAM), l'Association des Jeunes pour la Protection de l'Environnement et de l'Elevage (AJPEE), reconnue comme ONG et l'Association Zood Nooma pour le Développement (AZND) aussi reconnue comme ONG, le Projet Ecologique et de Reboisement (PER). Les artisans vitaux de ces regroupements sont les populations rurales, les structures d'appuis techniques et les bailleurs de fonds.

\section{Des alternatives à l'occupation des berges du lac \\ La libération de la bande de protection}

Selon les producteurs interrogés, des alternatives à l'occupation des berges du lac 
peuvent être envisagées. Ainsi, $75 \%$ des producteurs sont prêts à renoncer à l'occupation des berges à condition que d'autres propositions rentables leurs soient faites. S'il est établit que la majorité des producteurs est prête à renoncer à l'occupation des berges, les résultats de l'étude montrent que $65 \%$ ne sont pas prêts à changer d'activité même si un autre emploi leur est offert. Ils voudraient donc demeurer dans la pratique de leur activité initiale particulièrement le maraîchage. Ils souhaitent libérer les berges au moment où d'autres périmètres aménagés leur sont proposés. Mieux, parmi eux, 53\% sont disposés à payer de l'argent pour d'éventuels périmètres aménagés ailleurs. Par ailleurs, les 35\% sont favorables à une reconversion en cas de renonciation à l'occupation des berges.

\section{Des aménagements des périmètres irrigués}

Au regard des indicateurs ci-dessus, on retient que l'alternative qui se dégage est l'aménagement des périmètres irrigués au profit des populations riveraines. Mais les coûts d'installation sont souhaités être à la portée des populations car il ressort de l'enquête qu'elles peuvent payer en moyenne $35675 \pm 5000$ F CFA par hectare.

\section{La révision de la pratique de l'élevage}

Les résultats de l'enquête révèlent que l'élevage occupe une place de choix sur le lac chez 53\% des producteurs. D'ailleurs, cette activité constitue la deuxième source de revenu des populations. Sa révision pour une gestion efficace des berges va nécessiter la prise en compte d'un certain nombre de mesures d'accompagnement souhaitées par les populations. Il s'agit de l'aménagement d'abreuvoir pour éviter le piétinement des berges ainsi que la création de couloirs de pâturage afin d'éviter les conflits éleveursagriculteurs; la facilitation de l'accès à la ressource animale au profit des éleveurs.

\section{Reconversion dans d'autres activités}

Pour les personnes intéressées, cela va nécessiter des octrois de crédits. Ceci dans l'optique de faciliter leur installation. Mais l'activité aura besoin d'un suivi rigoureux pour s'assurer que les fonds sont bien utilisés. Les activités dans ce domaine sont la mécanique, l'installation de moulins, de petites boutiques de commerce général, des ateliers de coutures, etc.

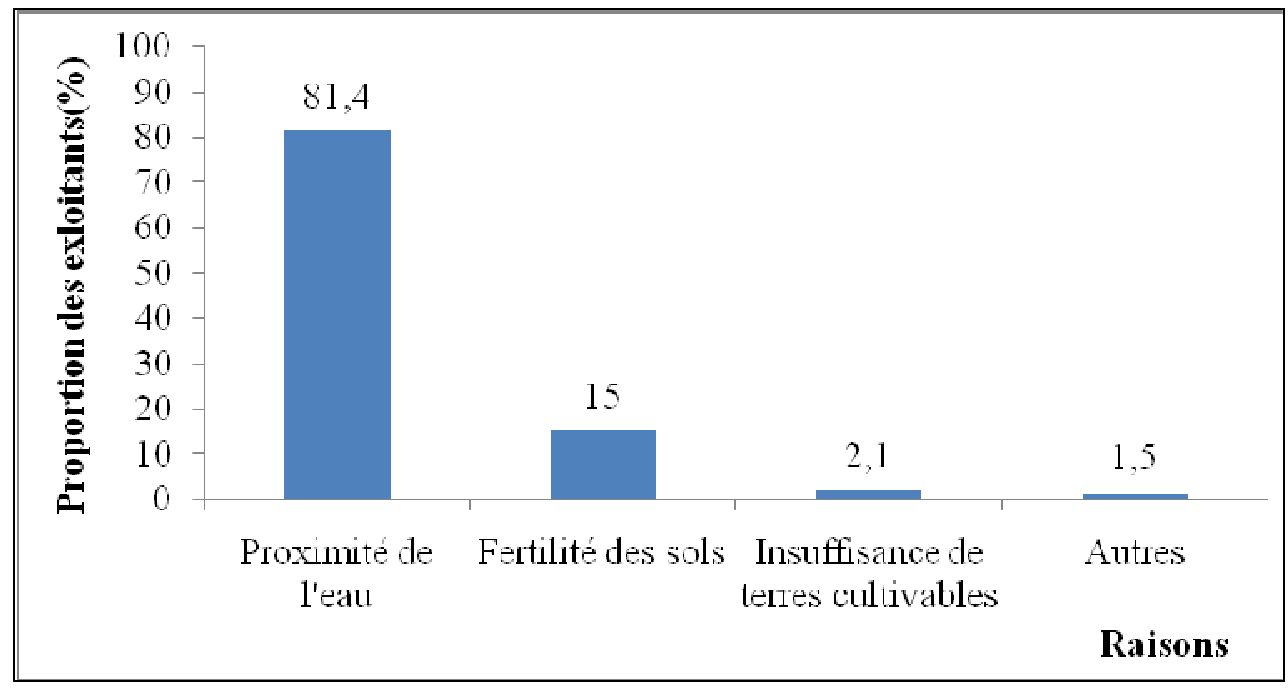

Figure 1: Les raisons de l'occupation des berges en fonction du pourcentage des exploitants. 
K. DRABO et al. / Int. J. Biol. Chem. Sci. 10(3): 944-956, 2016

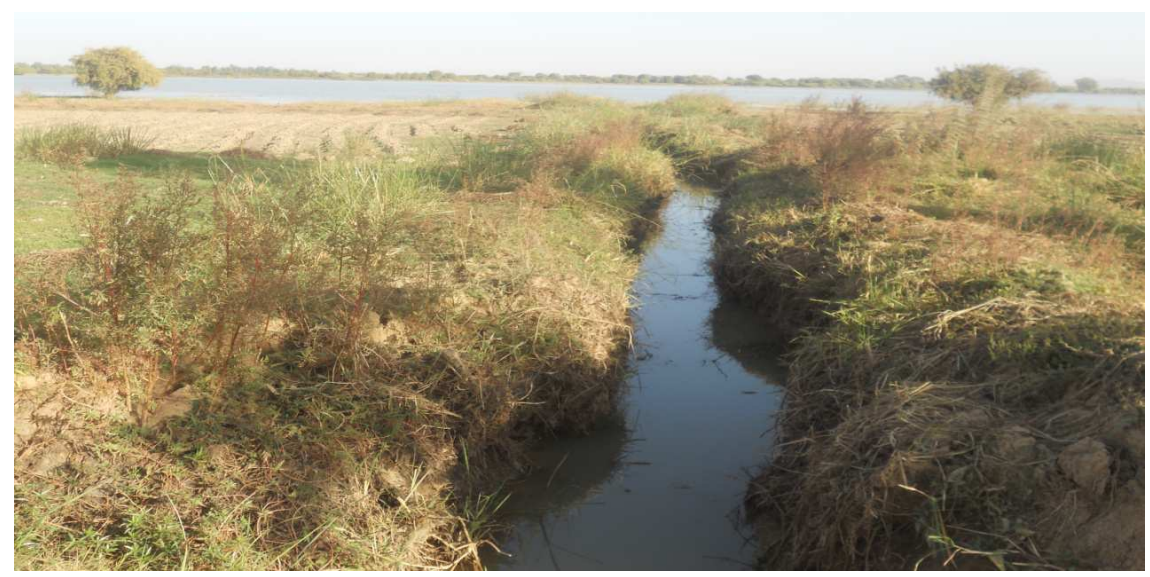

Figure 2 : Creusée de tranchée pour pompage d'eau.

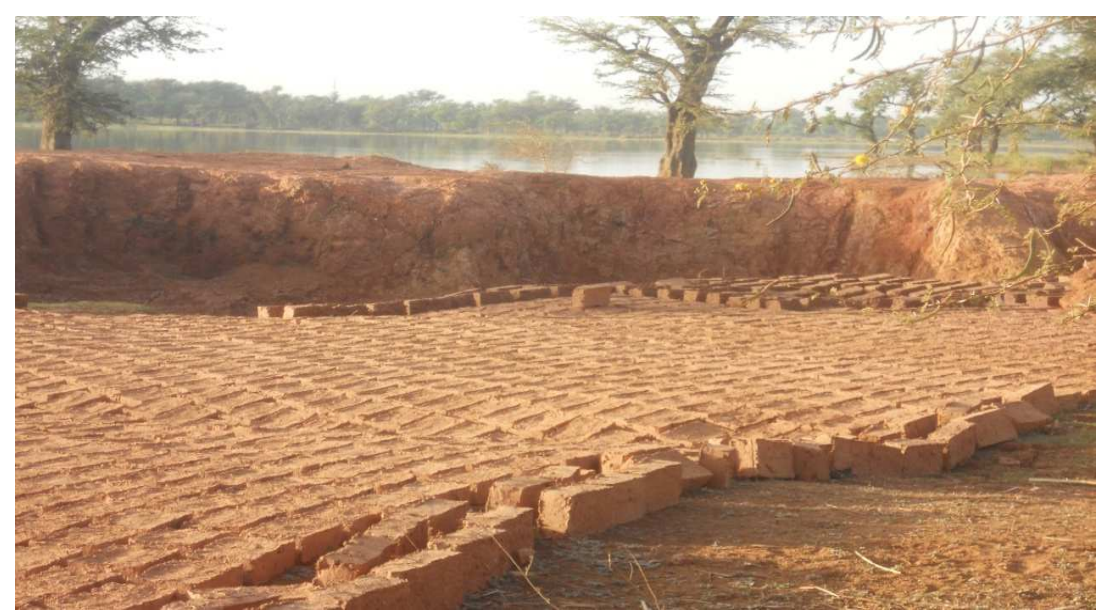

Figure 3 : Confection des briques autour du Lac Bam.

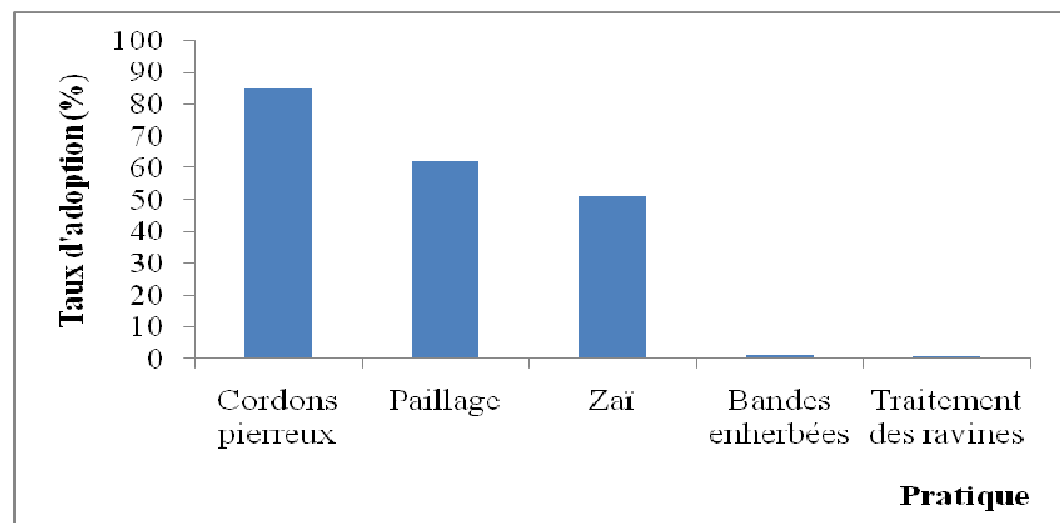

Figure 4 : Taux d'adoption des techniques de protection des berges du lac Bam par les producteurs. 
Tableau 1 : Les différents acteurs du Lac Bam, leurs missions, mérites et insuffisances.

\begin{tabular}{|c|c|c|c|}
\hline Acteurs & Missions / Actions & Mérites & Insuffisances \\
\hline $\begin{array}{l}\text { Populations riveraines } \\
\text { et personnes ressources } \\
\text { comme les chefs } \\
\text { coutumiers }\end{array}$ & $\begin{array}{l}\text {-Les populations riveraines sont impliquées à tous les } \\
\text { niveaux de l'exploitation et de la protection des } \\
\text { berges. } \\
\text {-Les chefs coutumiers jouent un rôle de propriétaires } \\
\text { terriens qui louent les terres aux producteurs. }\end{array}$ & $\begin{array}{l}\text {-L'étude révèle que les populations } \\
\text { riveraines ont une connaissance de } \\
\text { l'existence de la bande de servitude } \\
(87,1 \%)\end{array}$ & $\begin{array}{l}\text {-Les populations riveraines qui } \\
\text { devraient être impliquées à tous les } \\
\text { niveaux de la protection n'ont pas } \\
\text { suffisamment d'initiatives en matière } \\
\text { de protection du lac. } \\
\text {-Les locations de terres ne sont pas } \\
\text { assorties de code de bonne conduite en } \\
\text { matière de pratiques agricoles. Les } \\
\text { bénéficiaires des terres mènent leurs } \\
\text { activités sans un réel souci de } \\
\text { protection du lac. }\end{array}$ \\
\hline $\begin{array}{l}\text { Services techniques } \\
\text { déconcentrés de l'état }\end{array}$ & $\begin{array}{l}\text {-Définir la coordination, la conduite, la mise en } \\
\text { œuvre et le suivi technique des grandes orientations } \\
\text { politiques de gestion durable de l'environnement } \\
\text {-Participer à la gestion des conflits liés aux } \\
\text { ressources naturelles; } \\
\text {-Veiller au respect des lois et règlements des } \\
\text { politiques nationales et internationales en matière } \\
\text { d'eau, de la flore, de la faune, du sol, de l'élevage. }\end{array}$ & $\begin{array}{l}\text {-Ils ont une bonne connaissance des } \\
\text { mesures de protection dont ils ont en } \\
\text { charge, la mise en œuvre } \\
\text { - Ils accomplissent efficacement les } \\
\text { missions d'information, de } \\
\text { sensibilisation, de reboisement et } \\
\text { d'animation de cadres de concertations }\end{array}$ & $\begin{array}{l}\text {-Insuffisance financière et des } \\
\text { ressources humaines. Par exemple la } \\
\text { province du Bam dispose en tout dix } \\
\text { sept (17) forestiers et trois (03) } \\
\text { environnementalistes dont cinq }(05) \\
\text { forestiers pour la commune de } \\
\text { Kongoussi. }\end{array}$ \\
\hline $\begin{array}{l}\text { Associations et } \\
\text { groupements des } \\
\text { usagers du lac }\end{array}$ & $\begin{array}{l}\text {-Mènent des activités dans diverses filières, } \\
\text { maraîchages, élevage, pêche, Produits Forestiers Non } \\
\text { Ligneux et Ligneux }\end{array}$ & $\begin{array}{l}\text { Les membres mènent de nombreuses } \\
\text { actions de protection des berges avec } \\
\text { l'appui des Organisations Non } \\
\text { Gouvernementales (ONG) }\end{array}$ & $\begin{array}{l}93,6 \% \text { des membres de ces } \\
\text { associations et groupements ignorent } \\
\text { les textes réglementant la gestion des } \\
\text { berges ; } \\
\text {-insuffisances de moyens matériels } \\
\text { (vieilles motopompes) et financiers ; } \\
\text {-analphabétisme chez } 67,9 \% \text { des } \\
\text { membres ; }\end{array}$ \\
\hline
\end{tabular}


K. DRABO et al. / Int. J. Biol. Chem. Sci. 10(3): 944-956, 2016

\begin{tabular}{|c|c|c|c|}
\hline Acteurs & Missions / Actions & Mérites & Insuffisances \\
\hline $\begin{array}{l}\text { Les Organisations de la } \\
\text { société civile (OSC) }\end{array}$ & $\begin{array}{l}\text {-Défendre et préserver les intérêts des populations; } \\
\text {-Mener des actions remarquables sur le terrain en } \\
\text { matière de protection du lac et de ses ressources. } \\
\text { Leurs actions sont orientées entre autres, vers le } \\
\text { renforcement des capacités des producteurs; des } \\
\text { actions de conservation des eaux et la réhabilitation } \\
\text { des sols et des berges du lac; la lutte contre } \\
\text { l'ensablement du lac; la promotion de l'hygiène et } \\
\text { de l'assainissement etc. }\end{array}$ & $\begin{array}{l}\text {-Dynamisme remarquable de deux } \\
\text { organisations : Association des Jeunes } \\
\text { pour la Protection de l'Environnement } \\
\text { et de l'Elevage (AJPEE), reconnue } \\
\text { comme ONG de développement depuis } \\
\text { le } 15 \text { décembre } 1997 \text { et l'Association } \\
\text { Zood Nooma pour le Développement } \\
\text { (AZND) reconnue comme ONG } \\
\text { d'utilité publique depuis le } 23 \text { Août } \\
\text { 1996. }\end{array}$ & $\begin{array}{l}\text {-De nombreux obstacles de leadership, } \\
\text { financiers entravent toujours la } \\
\text { protection du lac. }\end{array}$ \\
\hline $\begin{array}{l}\text { Collectivités } \\
\text { Territoriales (CT) }\end{array}$ & $\begin{array}{l}\text { la loi } \mathrm{N}^{\circ} 055-2004 / \mathrm{AN} \text { du } 21 \text { décembre 2004, portant } \\
\text { Code Général des Collectivités Territoriales (CGCT) } \\
\text { confère aux collectivités territoriales l'autorisation } \\
\text { d'intervenir dans le domaine de l'environnement et } \\
\text { de la gestion des ressources naturelles. }\end{array}$ & $\begin{array}{l}\text { Les mesures de protection du lac sont } \\
\text { bien connues des différents services } \\
\text { techniques de la mairie }\end{array}$ & $\begin{array}{l}\text {-Les acteurs institutionnels des CT ne } \\
\text { sont pas reconnus et pris en compte } \\
\text { dans la loi d'orientation de gestion de } \\
\text { l'eau. } \\
\text {-Par conséquent les CT n'ont pas la } \\
\text { possibilité d'accomplir les missions qui } \\
\text { leur sont dévolues par le CGCT. } \\
\text { - depuis l'incident des populations } \\
\text { avec la société minière BISSA } \\
\text { GOLDE en 2011, la mairie a cessé de } \\
\text { se mêler des questions relatives au lac } \\
\text { Bam. }\end{array}$ \\
\hline
\end{tabular}




\section{DISCUSSION}

Les raisons de l'occupation des berges et les facteurs de dégradation interfèrent de façon étroite. En examinant les facteurs à l'origine de la dégradation des berges du Lac Bam, on note une défaillance par rapport à la vision de l'Etat burkinabé qui est de faire en sorte que la gestion des ressources naturelles prenne plusieurs contours en allant de la protection intégrale à la gestion décentralisée en passant par la cogestion par la trilogie EtatPopulations-Privé, ainsi que par la gestion associative et communautaire (Sawadogo, 2015). Les locations des terres non assorties de codes de bonne gestion; la non prise en compte des acteurs institutionnels des Collectivités Territoriales (CT) dans la loi d'orientation de la gestion de l'eau; la réticence des CT dans la résolution de certains problèmes de gestion du lac sont autant de facteurs qui fragilisent la synergie d'actions entre les trois entités Etat-Populations-Privés, synergie sans laquelle il serait difficile voire impossible de parvenir à des acquis pérennes (Tazen et al., 2013). Cette situation interpelle tout acteur, surtout les projets de développement au Bam de résoudre les questions relatives à ce mode de gestion. Mais comme le fait remarquer Traoré (2012) dans les sociétés rurales, la gestion communautaire ne produit pas que des effets positifs collectifs. Parfois, il faut discerner les confrontations entre les tendances communautaires et individualistes qui coexistent dans chaque localité.

Il importe aussi de reconnaître que le manque de moyens financiers sans cesse relevé par les populations et l'analphabétisme sont les plus grands maux qui minent le succès des projets de développement en milieu rural (SCADD, 2011). Des travaux dans la région du Centre-Nord ont révélé que la pauvreté des producteurs constitue une barrière redoutable aux applications des bonnes pratiques pour la gestion durable de l'environnement. Lorsque l'activité doit être mise en œuvre par des collectivités, elle connaît plus de succès que si elle doit être adoptée individuellement. Le manque de moyens pour l'adoption des bonnes pratiques entraînent la non adhésion des producteurs démunis. De même, l'analphabétisme est une barrière supplémentaire à la diffusion et à l'adoption des nouvelles technologies agricoles (Sp-CONEDD, 2008).

La raison principale de l'occupation des berges par les producteurs est la proximité de l'eau. Ainsi, préconiser leur éloignement de cette ressource c'est trouver des stratégies d'accéder à l'eau à distance sans une exigence considérable de frais et d'énergie de travail. Au niveau des berges, la stratégie prioritaire à réussir chez les populations riveraines demeure le retrait strict de leurs activités de la zone de protection. La non matérialisation de cette zone rend difficile les séances de sensibilisation et les différentes actions de répression qui pourraient être préconisées. Par ailleurs, il paraîtrait urgent aux marâichers représentant une forte proportion de disposer de motopompes pour les arrosages à distance afin de minimiser les problèmes communs de la pollution des eaux (Dovonou et al., 2011). Les propositions jusqu'ici faites ne répondent pas aux attentes des populations. L'Etat et ses partenaires financiers gagneraient le pari en rendant la motopompe (environ 500 000F l'unité) accessible aux producteurs. En matière de protection des berges les stratégies développées par le Projet Développement de l'Agriculture du Millenium Challenge Account Burkina Faso (MCA-BF) devraient servir d'exemples.

Si l'on considère la surexploitation des berges et qu'en matière d'alternatives à l'occupation des berges, une forte proportion des producteurs atteste demeurer dans la pratique du maraîchage, une solution envisageable est la création de nouveaux périmètres irrigués. Dans ce cas également, les réalisations du MCA-BF dans la Boucle du Mouhoun sont un signal fort pour l'Etat et ses partenaires (MCA-BF/AD10, 2011). Un nouveau périmètre irrigué implique un plan de gestion environnemental et social et un 
processus d'attribution des terres bien clarifiés (Hema, 2015). L'attribution des terres devrait être précédée de la détermination de la superficie minimale requise par producteur pour assurer la viabilité de l'exploitation.

En ce qui concerne les facteurs naturels, les observations des populations locales sont confirmées par la péjoration climatique (sécheresse et déficits hydriques chroniques) au Lac Bam qui se manifeste par l'augmentation des températures diurnes et entraîne une augmentation de l'évaporation et de l'évapotranspiration, d'où un risque d'assèchement du plan d'eau. Ces facteurs ont une incidence sur la qualité des sols. Les facteurs naturels de dégradations bien qu'ayant une envergure nationale et régionale $\mathrm{du}$ fait des changements climatiques, peuvent être minimisés par des pratiques de Conservation des Eaux et des Sols et de Défenses et Restauration des Sols (CESDRS). Les pratiques de cordons pierreux, de paillage et de zai sont largement vulgarisées dans les régions du Nord et du Centre-Nord (DMP/GEF, 2004) et les producteurs doivent constamment être incités à leurs mises en place et leur renouvellement.

Face aux menaces subies par le Lac Bam, nous pouvons faire les propositions suivantes: (i) matérialiser la bande de protection soit par des balises soit par des plantations d'arbres de grandes utilités (Deleke Koko et al., 2011) sur les limites et sur l'étendue de la surface afin de faciliter les séances de sensibilisation et les prises de sanctions contre les contrevenants aux mesures de protection; (ii) rendre accessible les motopompes et inspecter leur état d'amortissement. Cela permettra de lutter contre les pollutions des sols et de l'eau, d'éviter les nombreuses et fréquentes creusées de tranchées dans le seul but de se procurer de l'eau pour les activités agricoles; (iii) continuer à assurer des formations aux producteurs sur l'utilisations des herbicides et pesticides ; (iv) créer de nouveaux périmètres pour déconcentrer les producteurs sur les berges; Cette alternative nécessite la disponibilité de terres à aménager et la réalisation éventuelle de retenues d'eau à cette fin. L'aménagement doit prendre en compte le périmètre de protection des berges $(100 \mathrm{~m})$ conformément aux dispositions de la RAF (article 77) et élucider toute question relative au foncier même si la solution envisageable peut être l'expropriation de terres pour cause d'utilité publique suivie de dédommagement des intéressés; (v) dynamiser les cadres de concertations trilogique ci-dessus mentionné ; (vi) au niveau étatique, inciter les partenaires financiers à œuvrer à l'exemple du MCA-BF dans les régions de la Boucle du Mouhoun et des Cascades; (vii) la mise en place sur les berges, d'actions de démonstration de bonnes pratiques ou identifier des producteurs modèles à l'exemple du MCA-BF et du Programme d'Action National d'Adaptation (PANA) à la variabilité et aux changements climatiques. A cet effet, les acteurs de la recherche scientifique devraient être constamment sollicités. Toutefois, la recherche de meilleures conditions de production doit être couplée avec celle de l'écoulement des produits.

\section{Conclusion}

Les objectifs principalement poursuivis par ce travail ont abouti à l'identification d'une diversité de facteurs de dégradation naturels et anthropiques. Une faiblesse de synergie d'actions est perceptible entre les acteurs impliqués dans la gestion durable des berges du Lac Bam. Toute chose qui renforce les facteurs de dégradation des berges du Lac Bam. Les efforts restent à être consentis non seulement par les producteurs mais aussi par l'Etat et ses partenaires financiers et techniques afin de proposer des solutions idoines à la libération et à la protection des berges par les exploitants qui y sont déjà installés. Les solutions attendues concernent aussi bien les terres que l'équipement en matériel de travail. Des stratégies de mises en œuvre devraient conjuguer efficacement des approches individuelles et collectives pour 
une meilleure responsabilisation des acteurs à la protection des berges.

\section{CONFLIT D'INTERÊT}

Les auteurs déclarent qu'il n'y a aucun conflit d'intérêt dans le cadre de cet article.

\section{CONTRIBUTIONS DES AUTEURS}

KD a exécuté les travaux de terrain de la présente étude ; JY a orienté et supervisé les travaux de terrain ainsi que la rédaction de cet article; LS a contribué à la définition du thème et évalué la qualité des travaux.

\section{REMERCIEMENTS}

Les auteurs expriment leur gratitude aux agents des services publics en charge de l'eau, de l'environnement et des eaux et forets, de l'agriculture et de l'élevage ainsi que les autorités administratives pour leur disponibilité dans la conduite des travaux. Ils remercient les populations des villages de Kora, Darigma, Tanguiema, Pouni et Bam qui ont accompagné efficacement ces travaux.

\section{REFERENCES}

Badahoui E, Fiogbe ED, Boko M. 2009. Les causes de la degradation du chenal de Cotonou. Int. J. Biol. Chem. Sci., 3(5): 979-997. DOI : http://dx.doi.org/10.4314/ ijbcs.v3i5.51077

Deleke Koko IKE, Djego J, Gbenou J, Hounzangbe-Adote SM, Sinsin B. 2011. Etude phytochimique des principales plantes galactogènes et emménagogues utilisées dans les terroirs riverains de la Zone cynégétique de la Pendjari. Int. J. Biol. Chem. Sci., 5(2): 618-633. DOI : http://dx.doi.org/10.4314/ijbcs.v5i2.7212 7

DMP/GEF. 2004. Rapport d'Activités du Programme des Zones en Marges du Désert du Burkina Faso. CNRST-IRD report: Ouagadougou.

Dovonou F, Aina M, Boukari M, Alassane A. 2011. Pollution physico-chimique et bactériologique d'un écosystème aquatique et ses risques écotoxicologiques : cas du lac Nokoue au Sud Benin. Int. J. Biol. Chem. Sci., 5(4): 1590-1602.

DOI:

http://dx.doi.org/10.4314/ ijbcs.v5i4.23

Drabo K. 2015. Analyse des mesures de protection des berges du lac Bam au centre-nord Burkina Faso. Mémoire de Master II, Université Senghor, Campus Ouagadougou, p. 60.

Hema B. 2015. Analyse du processus d'attribution des terres mis en œuvre au profit des personnes affectées par le projet des villages de Di, Oué et Bouna dans la province du Sourou. Mémoire de Master II, 2iE, Ouagadougou, p. 64.

MCA-BF/AD10. 2011. Réinstallation sur le nouveau périmètre de Di. Livrable P15D du Millenium Challenge Account Burkina Faso: Ouagadougou, p. 81.

MEDD. 2014. Ministère de l'Environnement et du Développement Durable - Décret $\mathrm{N}^{\circ}$ 2014-069/PRES/PM/MEDD/MEF/ du 07 février 2014 portant adoption de la politique nationale sur les zones humides du Burkina Faso. JO $N^{\circ} 18$ du 1er Mai 2014, p. 32.

Sawadogo B. 2015. Analyse de l'état de la biodiversité de la forêt classée du site Ramsar de Dem au Burkina Faso Mémoire de Master II, Université Senghor, Campus Ouagadougou, Burkina Faso, p. 91.

Sawadogo LM. 2005. Impact des stratégies de lutte contre la dégradation des terres autour du lac Bam sur les conditions socioéconomiques des populations. Mémoire d'Ingénieur de l'Institut du Développement Rural, Université Polytechnique de Bobo-Dioulasso, BoboDioulasso, p. 66.

SCADD. 2011. Stratégie de Croissance Accélérée et de Développement Durable du Burkina Faso ( $1^{\text {ère }}$ edn). Ministère de l'Economie et des Finances Presse : Burkina Faso. 
SC-Ramsar. 2013. Manuel de la Convention de Ramsar du Secretariat Consultatif. $\left(6^{\mathrm{è}}\right.$ edn). SC-Ramsar : Suisse.

Smith J, Lavender B, Smit B, Burton I. 2001. Stratégies d'adaptation en vue de réduire la vulnérabilité des Canadiens aux changements climatiques, p.13.

Sp-CONEDD. 2008. Elaboration du document de projet PIF PPG du Programme d'Action National à la Variabilité et aux changements climatiques/Burkina Faso. Rapport sur la province du Namentenga et les villages pilotes. PANA report: Ouagadougou.

Tazen F, Fonteh MF, Karambiri H. 2013. Gestion intégrée des ressources en eau dans le bassin versant du lac municipal de Dschang : connaissance et usages. Int. J.
Biol. Chem. Sci., 7(2): 840-851. DOI: http://dx.doi.org/10.4314/ijbcs.v7i2.39

Thiombiano A, Kampmann D. 2010. Atlas de la Biodiversite de l'Afrique de l'Ouest. Tome II : Burkina Faso. (1ère edn) Ouagadougou et Francfort-sur-le-Main BIOTA : Ouagadougou.

Traoré R. 2012. Eau, territoire et conflits : analyse des enjeux de la gestion communautaire de l'eau au Burkina Faso : l'exemple du bassin versant du Nakambé. Thèse de doctorat unique, Université de Toulouse, France, p. 380.

Zoubga S. 2007. Burkina Faso. Le Réseau pour les Gommes et Résines Naturelles en Afrique (NGARA). NGARA Press: Ouagadougou. 